\title{
Sacral and thoracic chordoma with pulmonary metastases: A case report and review of the literature
}

\author{
RUI BAI*, ZHEN-QUN ZHAO*, YU-XIN WANG, WEI ZHAO, LI-SHUAN WU, \\ SHU-XIA CUI, SHI-BING GUO and CHEN-LIANG LIANG
}

Department of Orthopedic Surgery, Second Affiliated Hospital of Inner Mongolia Medical University, Hohhot, Inner Mongolia 010030, P.R. China

Received June 11, 2020; Accepted October 23, 2020

DOI: $10.3892 /$ mco.2020.2179

\begin{abstract}
Chordoma is a sporadic type of cancer that affects the spine and is particularly challenging to treat due to the paucity of reported cases and scientific literature. In particular, primary chordomas affecting both the sacral and thoracic vertebrae are extremely rare. We herein report a rare case of chordoma in the sacral and thoracic vertebrae with pulmonary metastasis, along with a literature review. The objective of the present study was to explore treatment options and long-term outcomes in patients with metastatic chordoma. Posterior decompression was performed for the thoracic tumor, followed by extended resection of the sacral tumor. The symptoms of the patient were relieved after surgery, and the postoperative Nurick score decreased from grade 3 to grade 2, while the postoperative McCormick score was I. Therefore, complete chordoma excision and internal spinal fixation may effectively reduce tumor recurrence and metastasis.
\end{abstract}

\section{Introduction}

Chordoma is a rare malignancy with an incidence rate of $0.8-5 / 100,000$ per year (1-4). Chordomas account for $1-4 \%$ of all bone malignancies and are predominant in men and in the 50-70 years age group $(1,5)$. Based on histological characteristics, chordomas may be subclassified into the typical, chondroid and dedifferentiated types. Chordomas arise from undifferentiated remnant cells of the embryonal notochord that reside within the axial skeleton (1). Although the mechanism underlying their malignant transformation is largely

Correspondence to: Dr Shi-Bing Guo or Dr Chen-Liang Liang, Department of Orthopedic Surgery, Second Affiliated Hospital of Inner Mongolia Medical University, 1 Yingfang Road, Wenhuagong Street, Huimin, Hohhot, Inner Mongolia 010030, P.R. China

E-mail: guoshibing2017@sina.com

E-mail:523984670@qq.com

*Contributed equally

Key words: chordoma, pulmonary metastases, sacral, thoracic unclear, mutations in the brachyury gene have been frequently detected in chordoma patients (6). Furthermore, 50-60\% of the tumors occur in the sacrococcygeal region, $25-35 \%$ at the skull base and $10-15 \%$ in the mobile spine (7). The clinical manifestations of chordoma depend on tumor location and invasion, and typically include radiculopathy, deeply localized pain and neurological deficits. Computed tomography (CT) and magnetic resonance imaging (MRI) are the preferred methods for determining chordoma extension into the bone and adjacent soft tissues. The tumor masses appear as lytic lesions and may contain areas of calcification on CT scans, and as iso- to hypointense on T1-weighted MRI and hyperintense on T2-weighted MRI $(1,5)$. The most effective treatment is surgical excision, although it is technically challenging due to the complex anatomical structure of the affected sites. Therefore, postoperative complications and recurrence are common, and the local recurrence rate is as high as $43-85 \%$ (8), whereas only $5 \%$ of the tumors metastasize, typically to the lung, bone or brain. Pulmonary metastasis of primary sacral and thoracic chordomas is rare. The aim of the present study was to describe a rare case of sacral and thoracic vertebral chordoma with pulmonary metastasis, and to review the available literature on chordomas of the thoracolumbar spine.

\section{Case report}

A 73-year-old man presented at the Department of Orthopedic Surgery of the Second Affiliated Hospital of Inner Mongolia Medical University in June 2018 with intermittent lumbosacral pain and root pain of the lower limbs, which severely affected his gait and gradually led to difficulty in defecating and urinating. On inspection and palpation, a large soft tissue mass was identified in the lumbosacral area. The patient had been manifesting the symptoms for 8 years, and the lower back pain had progressed in the preceding 2 years. The patient had also undergone rectostomy and indwelling catheterization for incontinence 2 years prior. The preoperative Nurick grade and McCormick score were 3 and II, respectively. Thoracolumbar CT and MRI examinations revealed bone destruction of the right medial wall of the spinal canal and right pedicle of the T11 vertebra, protrusion of the soft tissue mass into the spinal canal, with dural sac compression and shift to the left (Fig. 1). In addition, bone dissolution was observed below the S2 level, 
along with a $14 \times 13 \times 12.6-\mathrm{cm}$ round soft tissue mass with a clear boundary, and an irregular calcified spot (Fig. 2A-D). Pelvic angiography revealed abundant blood supply to the tumor (Fig. 2E). Furthermore, pulmonary CT scan revealed multiple dense nodules with a smooth border, with a maximum diameter of $\sim 0.95 \mathrm{~cm}$, in both lungs (Fig. $2 \mathrm{~F}$ ). The patient also presented with hypertension, first-degree atrioventricular block and cerebral infarction sequelae. Based on these findings, a diagnostic biopsy was recommended.

Sacral needle biopsy, hematoxylin-eosin and immunohistochemical staining confirmed the diagnosis of chordoma positive for AE1/AE3, S100 and cytokeratin (CK)8/18 expression (Fig. 3A-C). Accordingly, the tumor mass in the thoracic vertebrae was considered to be metastatic chordoma. However, due to the risk of spinal cord injury, puncture biopsies of the thoracic vertebral tumor were not performed. Posterior laminectomy and tumor curettage were used on the thoracic vertebrae (Fig. 4A and B). On histopathological examination, the T11 vertebral specimen exhibited cytoplasmic vacuolation (Fig. 3D), and was positive for epithelial membrane antigen and CK19 (Fig. 3E and F), and negative for CK7 and chromogranin A expression, findings that were indicative of chordoma.

The posterior median spinous process of the lumbosacral spine (L3-S1, $20 \mathrm{~cm}$ ) were removed, and the L4-S1 bilateral lamina, superior and inferior articular processes, bilateral iliac wings and positioning needle were exposed. Four pedicle screws were fastened into both sides of L4 and L5. An inverted ' $\mathrm{Y}$ ' incision was extended to the distal end to reveal the posterior sacrococcyx and sacroiliac joint, and the sacral mass was extensively resected. Two iliac screws were fastened downwards on both sides at the S1 level, and the connecting rods were installed on the left and right sides, followed by locking and fixing the nuts (Fig. 4C-G). The volume of intraoperative blood loss was $\sim 3,000 \mathrm{ml}$. The patient did not receive any chemotherapy or radiotherapy after surgery, and the pulmonary X-ray at the 24-month follow-up revealed stable disease (Fig. 5A). In addition, X-ray and CT examination revealed stable internal fixation of the spine, and there was no clinical or radiological evidence of local recurrence or metastasis (Fig. 5B-F). The patient achieved good spine stabilization and was able to walk and perform daily activities without assistance. The postoperative Nurick score decreased from grade 3 to grade 2, and the postoperative McCormick grade was I. On the last follow-up (June 2020) the patient was in good overall condition with good mobility, and the lung lesions were stable. Computed tomography reconstruction of the thoracic vertebra and pelvis revealed no loosening or fracture in the internal fixation, and no local tumor recurrence.

\section{Discussion}

Chordomas are slow-growing, low-to-intermediate-grade tumors that are often diagnosed at an advanced stage with significant bony destruction and soft tissue invasion $(1,5)$. Chordomas may be falsely diagnosed as relatively benign tumors, and their resection may be challenging due to their high invasiveness. Therefore, chordoma resection is associated with a high risk of morbidity and mortality. In addition, the epidemiology of chordoma is unclear (9), particularly as regards its incidence rates and the most frequently affected parts of the axial skeleton $(10,11)$. This is clinically significant, since the symptoms and treatment strategies of chordoma largely depend on its anatomical location $(12,13)$.

Pain is the most common symptom of chordoma. Furthermore, spinal cord or nerve root compression may result in neurological symptoms, whereas nerve root involvement in the sacrum leads to bowel and bladder dysfunction, including incontinence (14). Although CT and MRI may be used to determine the extent of chordoma lesions and facilitate surgical planning, pathological examination following puncture biopsy is the gold standard for diagnosing chordoma. Complete surgical resection with negative margins may effectively reduce the risk of local recurrence and distant metastasis $(15,16)$. Our patient had been experiencing intermittent lumbosacral pain and root pain of the lower limbs over the previous 2 years, and eventually developed incontinence. CT and MRI examination revealed bone destruction of the right medial wall of the spinal canal and right pedicle of the T11 vertebra, and a soft tissue mass with well-defined boundaries protruding into the spinal canal, resulting in dural sac compression. Therefore, posterior decompression of the thoracic tumor was performed to reduce compression symptoms, and the spine was stabilized by intravertebral fixation, followed by extensive sacral tumor resection.

The 2020 NCCN guidelines (17) for the diagnosis and treatment of sacrococcygeal or spinal chordomas recommend extensive resection of operable lesions. Postoperative radiotherapy may be considered if the resection margin is positive or if the soft tissue mass is large ( $>7 \mathrm{~cm}$ in largest diameter). Surgical resection, whether curative or subtotal, remains the gold standard for the treatment of chordoma. It has been reported that the recurrence rate of chordomas located in the sacrum and spine was $33 \%$ following extensive resection and $67 \%$ after intracapsular or marginal resection (18). Sacral chordomas may be classified into proximal and distal types, depending on the affected segments; the former involves the first and second segments of the sacrum, and the latter originates from other segments. A combined anteroposterior approach is considered as appropriate for proximal sacrum chordoma and the posterior approach for distal sacrum chordoma (19). For S1 or S2 level tumors, resection via the posterior approach is considered as adequate and is associated with an acceptable risk of surgical complications. However, curative or complete resection is difficult to achieve due to invasion of the tumor mass into the vertebral body, spinal cord, nerve roots, peripheral nerves, or other tissues. Therefore, only 10-20\% of chordomas are curable by surgery, and the postoperative recurrence rate is high (20).

Radiotherapy is often combined with surgery to improve local control. A recent retrospective study reported that the 5-year local control and survival rates were 85.4 and $81.9 \%$, respectively, in patients without surgical resection of chordoma who were treated by photon or proton radiotherapy (21). A chordoma recurrence rate of $>90 \%$ has been reported following subtotal intratumoral resection and radiotherapy, which may be attributed to incomplete tumor removal (22). By contrast, Park et al demonstrated that local radiotherapy after complete resection improved the 5- and 10-year survival rates to 93 and 91\%, respectively (23). For patients who are not candidates for surgical intervention due to underlying conditions 
A
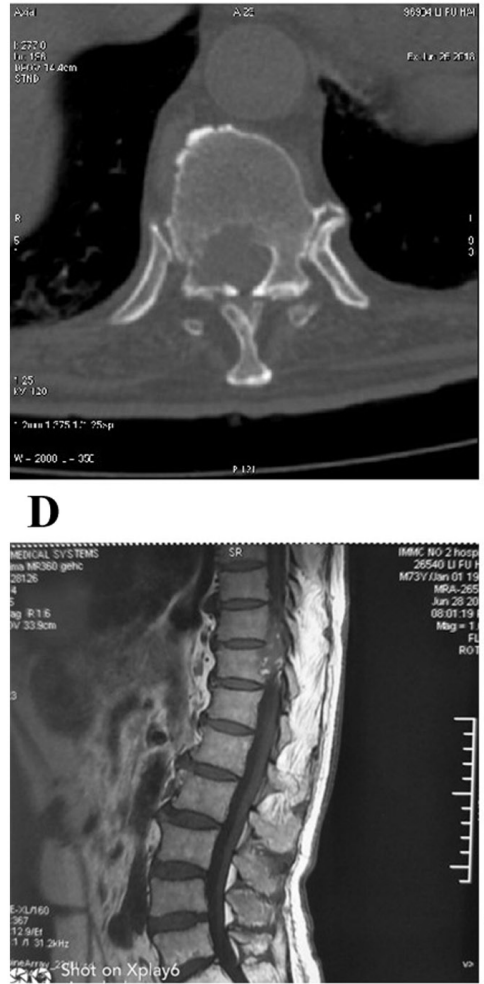

B

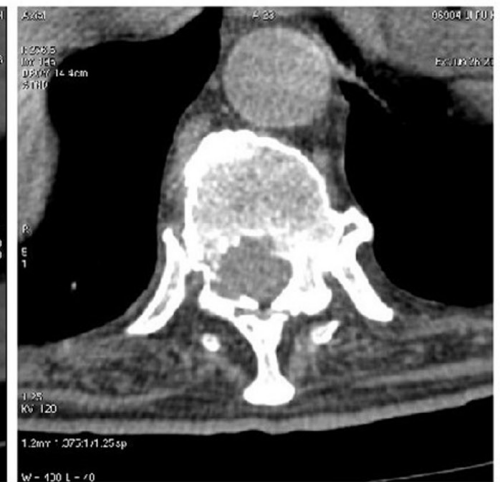

$\mathbf{E}$

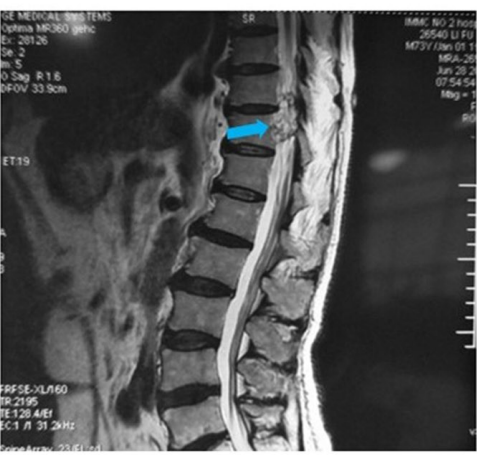

C

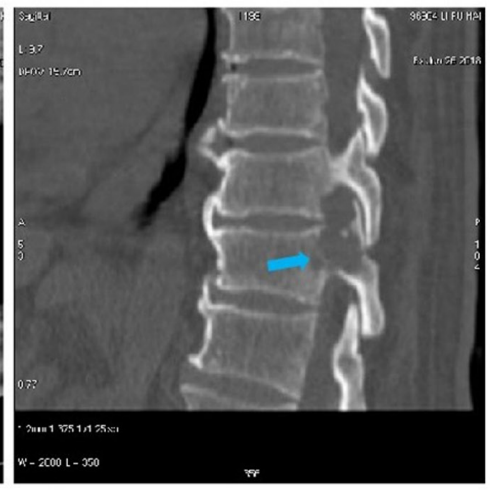

$\mathbf{F}$

Figure 1. (A-C) Computed tomography and (D-F) magnetic resonance imaging showing bone destruction of the right medial wall of the spinal canal and right pedicle of T11 vertebra, soft tissue mass protrusion into the spinal canal, and dural sac compression and shift to the left (arrows).

A

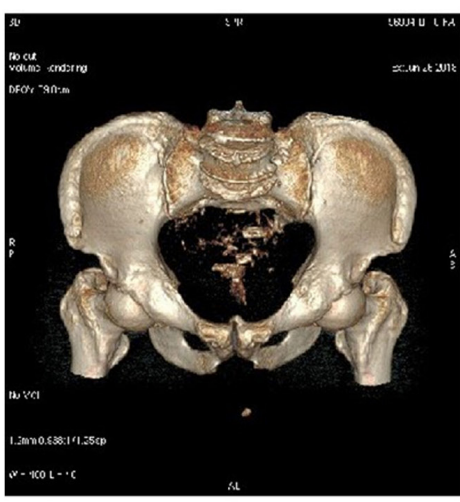

D

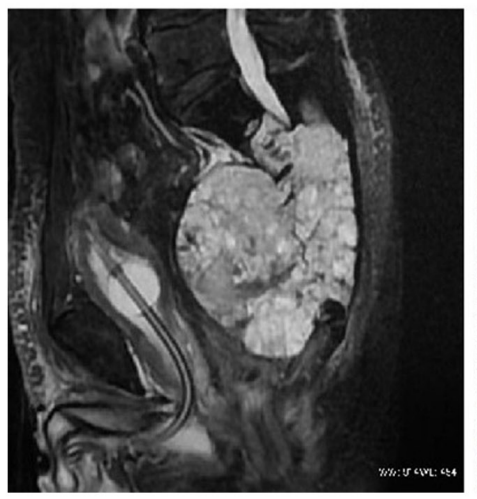

B

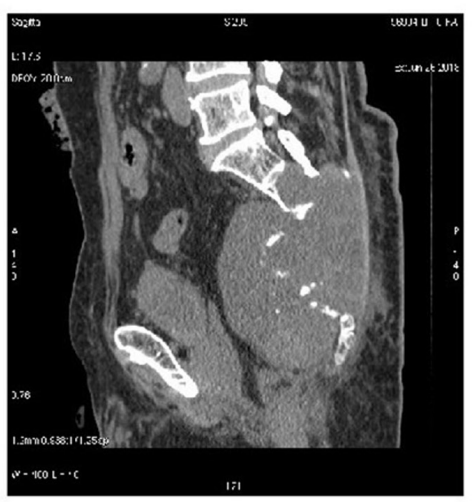

$\mathbf{E}$

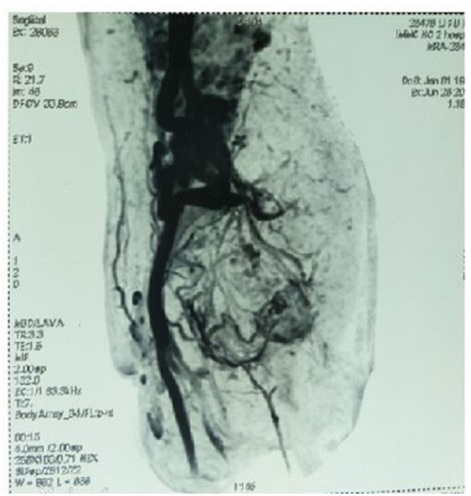

C

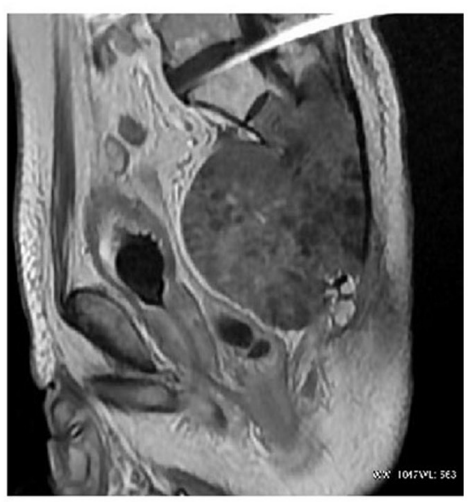

$\mathbf{F}$

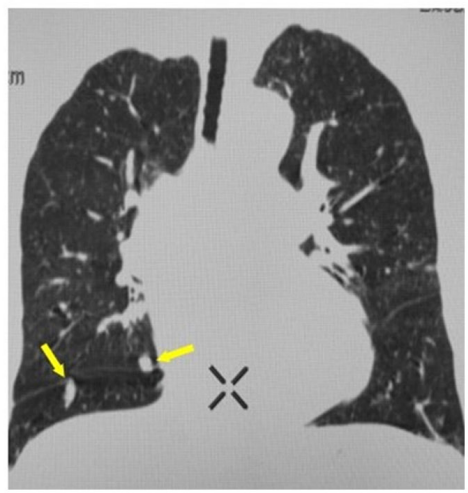

Figure 2. (A-D) Computed tomography scan and magnetic resonance imaging showing bone dissolution and destruction below the $\mathrm{S} 2$ level, and a 14x13x12.6-cm round soft tissue mass with a clear boundary and irregular spot-like calcification. (E) Pelvic angiography revealed abundant blood supply to the tumor. (F) Computed tomography scan of both lungs showing multiple dense pulmonary metastases with a smooth border, with a maximum diameter of $\sim 0.95 \mathrm{~cm}$ (arrows). 
A

B

C
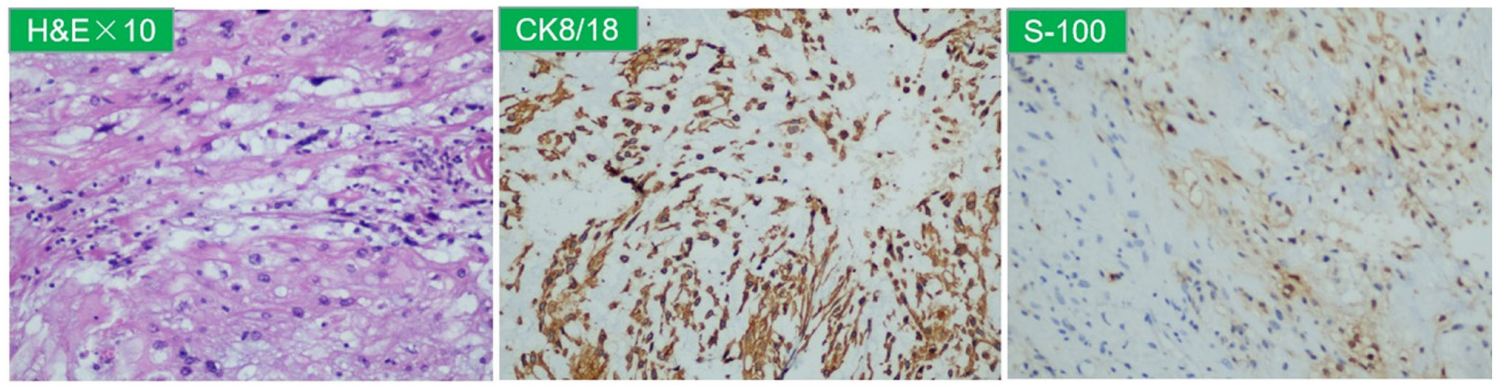

D

E

F
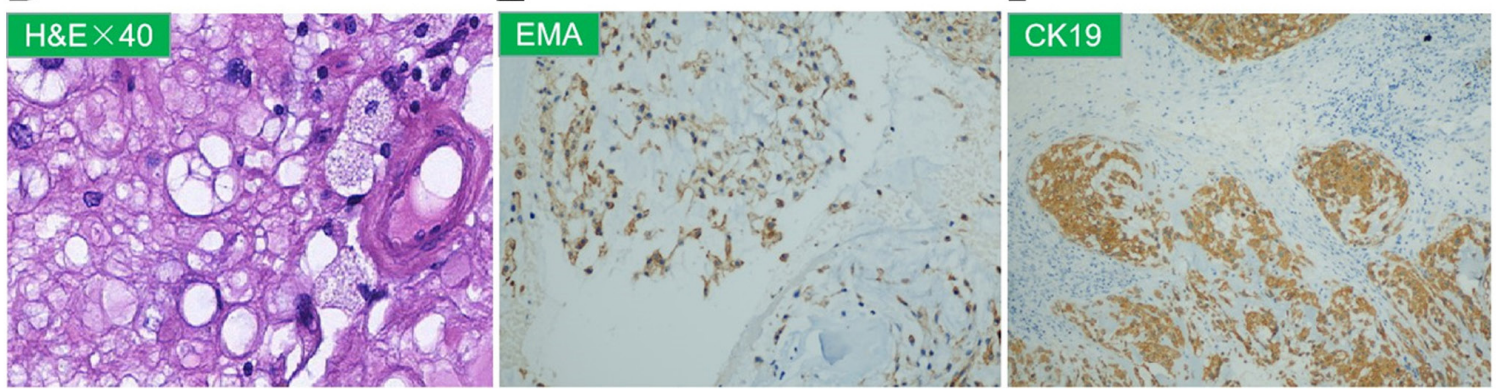

Figure 3. (A-F) Sacral needle core biopsy of the T11 vertebra. (A and D) Representative images of H\&E-stained sections (magnification, $\mathrm{x} 10$ and $\mathrm{x} 40$, respectively) showing cytoplasmic vacuolation. Representative images of immunohistochemical staining for (B) CK8/18, (C) S-100, (E) EMA and (F) CK19. $\mathrm{H} \& \mathrm{E}$, hematoxylin and eosin; CK, cytokeratin; EMA, epithelial membrane antigen.

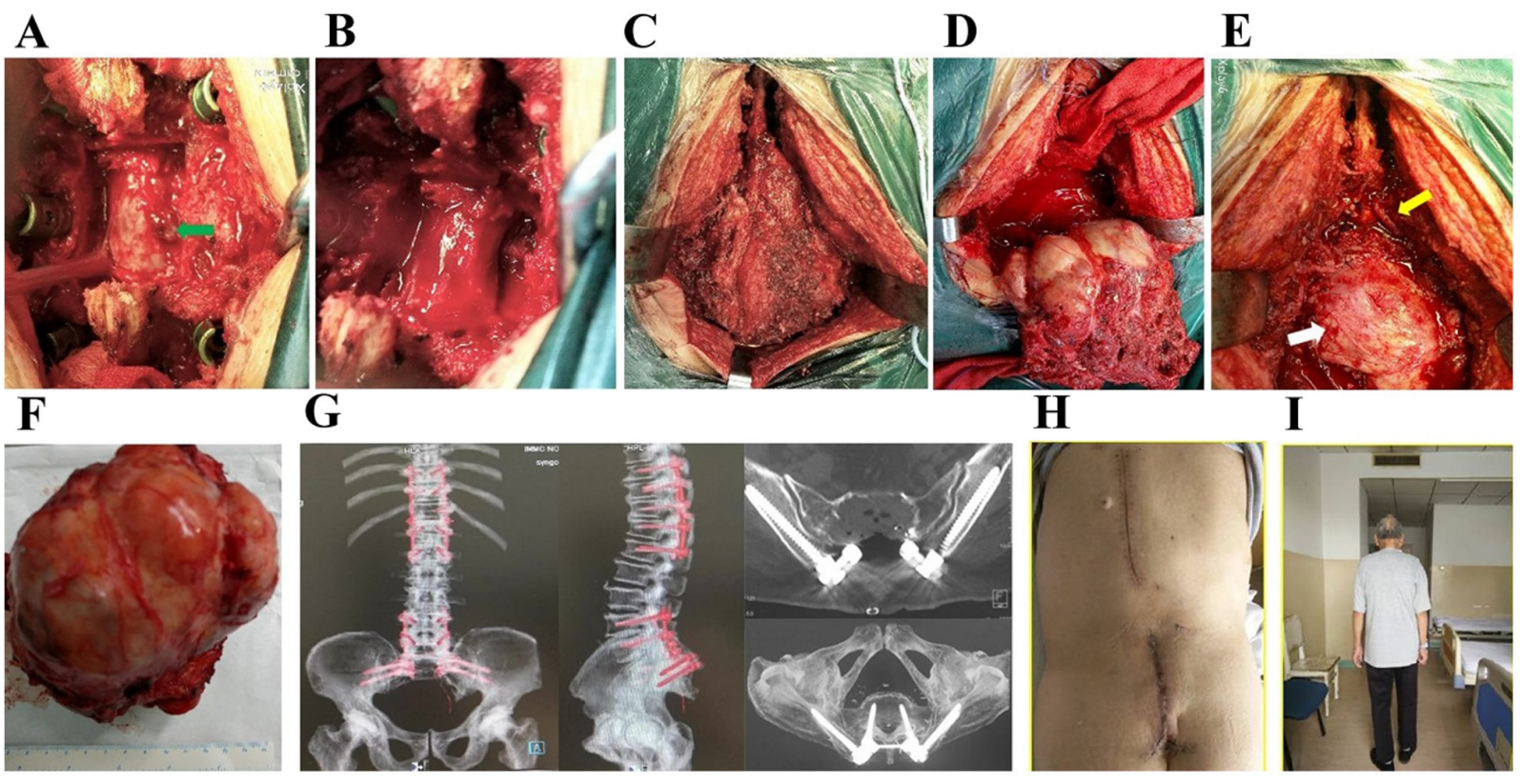

Figure 4. (A and B) Stage I resection of the thoracic intraspinal mass protruding into the spinal canal (arrow) after posterior decompression. (C and D) Stage II extended (C) sacral tumor resection with (D) removal of S1 and S2 spaces of the upper boundary of the tumor. (E) The S1 nerve (yellow arrow) and the posterior peritoneum of the rectum (white arrow) remained intact after surgery. (F) The resected tumor measured $\sim 14 \times 15 \mathrm{~cm}$. (G) Computed tomography with maximum intensity projection image reconstruction after posterior thoracic decompression with screw-rod internal fixation and sacral tumor resection. (H) Inspection of incision healing after surgery. (I) Walking ability was restored 2 weeks after surgery.

(e.g., compromised renal/cardiac function), radiation therapy alone may be warranted. Chen et al (24) reported a 5-year survival rate of $78 \%$ with high-dose radiation in a study on patients with chordoma who were not deemed fit for surgery. Therefore, an initial tumor debulking surgery followed by radiotherapy and chemotherapy, may be a viable strategy. In addition, radiotherapy may also be used for inoperable patients.
There is currently no clearly effective drug recommended for the systemic treatment of chordoma. Imatinib and sorafenib may delay disease progression or partially alleviate symptoms in some cases (25-29). Erlotinib and apatinib may also be administered $(30,31)$. However, conventional chemotherapy is not considered as a suitable option for advanced chordomas, apart from cases with well-differentiated tumors (32-34). 
A

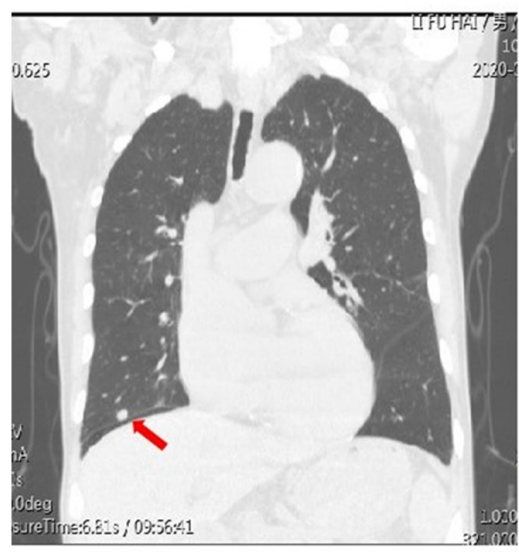

D

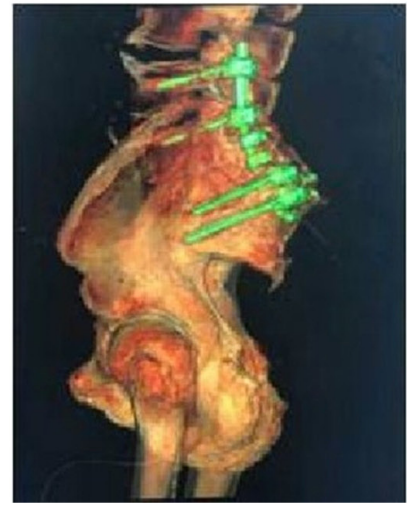

B

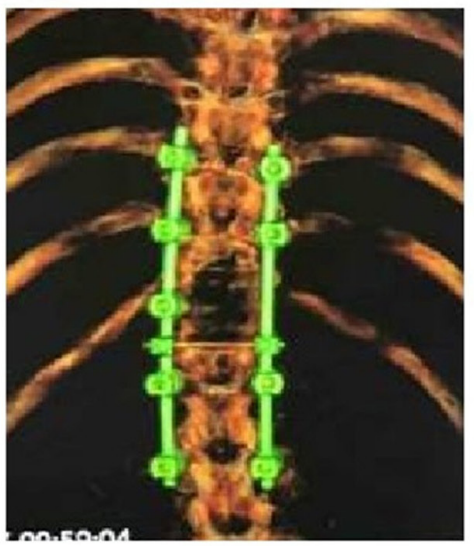

C

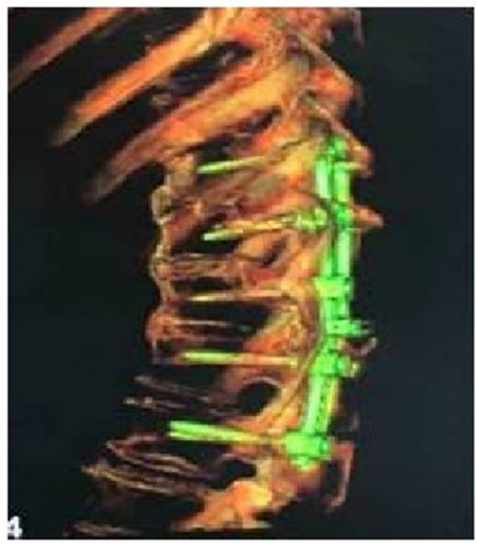

$\mathbf{E}$

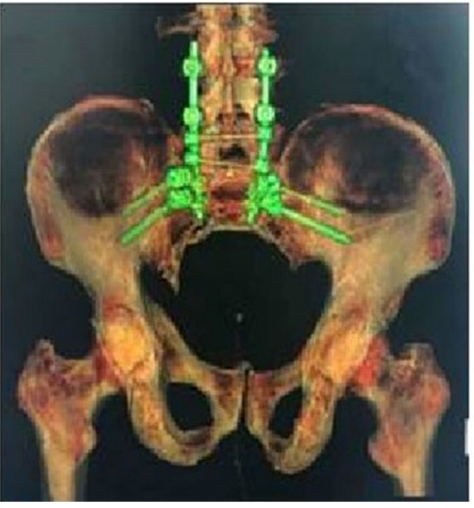

F

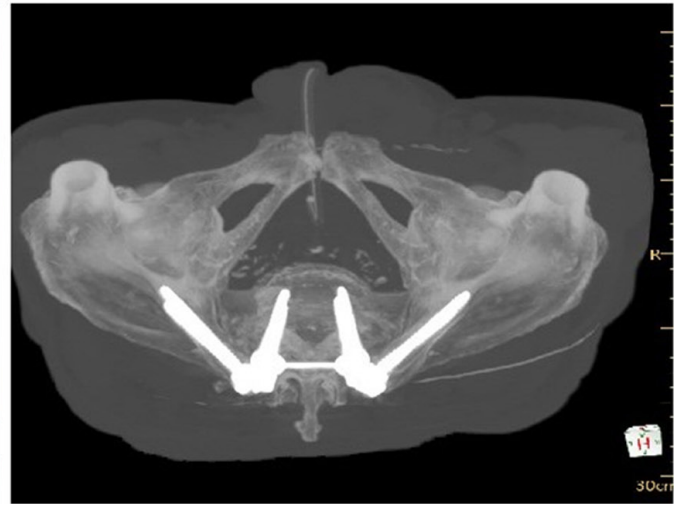

Figure 5. (A) Stable lung lesions (arrow) after surgery. (B-F) Computed tomography reconstruction of the thoracic vertebra and pelvis showed no loosening or fracture in the internal fixation, and no local tumor recurrence.

Despite the low risk $(5 \%)$ of metastasis, the prognosis of chordoma remains poor, with 5- and 10-year survival rates of $50-68$ and $28-40 \%$, respectively (5). A retrospective analysis of 115 patients with sacral chordoma who underwent surgery revealed a 5 -year overall survival rate of $81 \%$ over a mean follow-up duration of 4.9 years (range, 1.3-10.8 years). The survival rates declined in the initial 3 years and improved in the 4th year. The effect of surgical margin and prior surgery were not correlated linearly with conditional survival (35). Another retrospective study on 171 patients with sacral chordoma recurrence indicated metastasis in 17 cases $(10.8 \%)$, including 5 with primary pulmonary metastasis, 3 with bone metastasis (ribs and sternum) and 9 with postoperative pulmonary metastasis. Of the 14 patients with pulmonary metastases, 9 succumbed to the disease, whereas 2 patients with rib metastases underwent rib resection and did not develop any new lesions. A patient with spinal metastasis underwent tumor removal and decompression, followed by a second surgery upon recurred in the spine. Furthermore, local recurrence was identified as the only significant risk factor for metastasis (36).

Based on the findings of the present case and those of previous studies, it may be inferred that local progression and recurrence are the most crucial prognostic factors of chordoma. In addition, the extent of the initial surgical resection is also prognostically significant. It was previously reported that age, sex, treatment history, tumor location, pathological grade, surgical margin, radiotherapy and chemotherapy affect the long-term prognosis of chordoma patients (37). In the same cohort, younger age (pediatric group), sacral location, dedifferentiated tumors and chemotherapy were identified as independent risk factors of shorter progression-free survival, whereas larger tumor size and tumor necrosis have also been implicated as negative prognostic factors (7).

Lumbosacral chordoma with pulmonary metastasis is difficult to treat due to its anatomical location and high risk of local recurrence. The case presented herein demonstrated that radical surgery may effectively control the local recurrence or distant metastasis of chordoma, whereas the combination of surgery and postoperative adjuvant therapy may further improve patient outcomes.

\section{Acknowledgements}

Not applicable.

\section{Funding}

No funding was received.

\section{Availability of data and materials}

All data generated or analyzed in the present study are included in this published article. 


\section{Authors' contributions}

SBG and RB conceived and designed the study. SBG, RB, ZQZ, YXW, WZ and CLL participated in the design of the study and drafting of the manuscript. LSW and SXC performed anesthesia and monitoring for surgery. SBG, RB, ZQZ, CLL, YXW and WZ reviewed and edited the manuscript. All the authors have read and approved the final version of the manuscript.

\section{Ethics approval and consent to participate}

The study was approved by the Ethics Committee of the Second Affiliated Hospital of Inner Mongolia Medical University.

\section{Patient consent for publication}

Written informed consent was obtained from the patient for the publication of the case details and associated images. A copy of the consent form is available for review.

\section{Competing interests}

All the authors declare that they have no competing interests.

\section{References}

1. Walcott BP, Nahed BV, Mohyeldin A, Coumans JV, Kahle KT and Ferreira MJ: Chordoma: Current concepts, management, and future directions. Lancet Oncol 13: e69-e76, 2012.

2. Ropper AE, Cahill KS, Hanna JW, McCarthy EF, Gokaslan ZL and Chi JH: Primary vertebral tumors: A review of epidemiologic, histological and imaging findings, part II: Locally aggressive and malignant tumors. Neurosurgery 70: 211-219, 2012.

3. Chambers KJ, Lin DT, Meier J, Remenschneider A, Herr M and Gray ST: Incidence and survival patterns of cranial chordoma in the United States. Laryngoscope 124: 1097-1102, 2014.

4. McMaster ML, Goldstein AM, Bromley CM, Ishibe $\mathrm{N}$ and Parry DM: Chordoma: Incidence and survival patterns in the United States, 1973-1995. Cancer Causes Control 12: 1-11, 2001.

5. Sciubba DM, Chi JH, Rhines LD and Gokaslan ZL: Chordoma of the spinal column. Neurosurg Clin N Am 19: 5-15, 2008.

6. Jahangiri A, Jian B, Miller L, El-Sayed IH and Aghi MK: Skull base chordomas: Clinical features, prognostic factors, and therapeutics. Neurosurg Clin N Am 24: 79-88, 2013.

7. Chugh R, Tawbi H, Lucas DR, Biermann JS, Schuetze SM and Baker LH: Chordoma: The non sarcoma primary bone tumor. Oncologist 12: 1344-1350, 2007.

8. Fuchs B, Dickey ID, Yaszemski MJ, Inwards CY and Sim FH: Operative management of sacral chordoma. J Bone Joint Surg Am 87: 2211-2216, 2005

9. Bongers MER, Dea N, Ames CP and Schwab JH: Surgical strategies for chordoma. Neurosurg Clin N Am 31: 251-261, 2020.

10. D'Amore T, Boyce B and Mesfin A: Chordoma of the mobile spine and sacrum: Clinical management and prognosis. J Spine Surg 4: 546-552, 2018.

11. Alan O, Akin Telli T, Ercelep O, Tanrikulu Simsek E, Basoglu Tuylu T, Mutis A, Hasanov R, Kaya S, Akgül Babacan N, Dane F and Yumuk PF: Chordoma: A case series and review of the literature. J Med Case Rep 12: 239, 2018.

12. Vellutini EAS, Brock RS, Martins HO, Taricco MA and de Oliveira MF: Diffuse spinal spreading following previous intracranial intradural chordoma resection: A rare case report. J Clin Neurosci 64: 44-46, 2019.

13. Akmansu M, Kurt G, Demircan V and Senturk E: Results of chordoma patients treated by different approaches in a single institution. Turk Neurosurg 30: 366-370, 2020.
14. Ahmed R, Sheybani A, Menezes AH, Buatti JM and Hitchon PW: Disease outcomes for skull base and spinal chordomas: A single center experience. Clin Neurol Neurosurg 130: 67-73, 2015.

15. Pu F, Wang B, Liu J, Chen F and Shao Z: Giant chordoma in the thoracolumbar spine: A case report and literature review. Eur Spine J 26 (Suppl 1): S95-S99, 2017.

16. Colangeli S, Muratori F, Bettini L, Frenos F, Totti F, D'Arienzo A, Campo FR, Scoccianti G, Beltrami G, Campanacci DA and Capanna R: Surgical treatment of sacral chordoma: En bloc resection with negative margins is a determinant of the long-term outcome. Surg Technol Int 33: 343-348, 2018.

17. Niu XH: Interpretation of 2020 NCCN clinical practice guidelines in oncology-bone cancer. Zhonghua Wai Ke Za Zhi 58: 430-434, 2020 (In Chinese).

18. Ruggieri P, Angelini A, Ussia G, Montalti M and Mercuri M: Surgical margins and local control in resection of sacral chordomas. Clin Orthop Relat Res 468: 2939-2947, 2010.

19. Mohanty S, Pai Kanhangad M and Kundangar R: The extended posterior approach for resection of sacral tumours. Eur Spine J 28: 1461-1467, 2019.

20. Yang Y, Li Y, Liu W, Xu H and Niu X: The clinical outcome of recurrent sacral chordoma with further surgical treatment. Medicine (Baltimore) 97: e13730, 2018.

21. Kabolizadeh P, Chen YL, Liebsch N, Hornicek FJ, Schwab JH, Choy E, Rosenthal DI, Niemierko A and DeLaney TF: Updated outcome and analysis of tumor response in mobile spine and sacral chordoma treated with definitive high-dose photon/proton radiation therapy. Int J Radiat Oncol Biol Phys 97: 254-262, 2017.

22. Imai R, Kamada T, Tsuji H, Sugawara S, Serizawa I, Tsujii H and Tatezaki S; Working Group for Bone and Soft Tissue Sarcomas: Effect of carbon ion radiotherapy for sacral chordoma: Results of Phase I-II and Phase II clinical trials. Int J Radiat Oncol Biol Phys 77: 1470-1476, 2010.

23. Park L, Delaney TF, Liebsch NJ, Hornicek FJ, Goldberg S, Mankin H, Rosenberg AE, Rosenthal DI and Suit HD: Sacral chordomas: Impact of high-dose proton/photon-beam radiation therapy combined with or without surgery for primary versus recurrent tumor. Int J Radiat Oncol Biol Phys 65: 1514-1521, 2006.

24. Chen YL, Liebsch N, Kobayashi W, Goldberg S, Kirsch D, Calkins G, Childs S, Schwab J, Hornicek F and DeLaney T: Definitive high-dose photon/proton radiotherapy for unresected mobile spine and sacral chordomas. Spine (Phila Pa 1976) 38: E930-E936, 2013.

25. Bompas E, Le Cesne A, Tresch-Bruneel E, Lebellec L, Laurence V, Collard O, Saada-Bouzid E, Isambert N, Blay JY, Amela EY, et al: Sorafenib in patients with locally advanced and metastatic chordomas: a phase II trial of the French Sarcoma Group (GSF/GETO). Ann Oncol 26: 2168-2173, 2015.

26. Lebellec L, Bertucci F, Tresch-Bruneel E, Bompas E, Toiron Y, Camoin L, Mir O, Laurence V, Clisant S, Decoupigny E, et al: Circulating vascular endothelial growth factor (VEGF) as predictive factor of progression-free survival in patients with advanced chordoma receiving sorafenib: An analysis from a phase II trial of the French sarcoma group (GSF/GETO). Oncotarget 7: 73984-73994, 2016.

27. Stacchiotti S, Longhi A, Ferraresi V, Grignani G, Comandone A, Stupp R, Bertuzzi A, Tamborini E, Pilotti S, Messina A, et al: Phase II study of imatinib in advanced chordoma. J Clin Oncol 30: 914-920, 2012.

28. George S, Merriam P, Maki RG, Van den Abbeele AD, Yap JT, Akhurst T, Harmon DC, Bhuchar G, O'Mara MM, D'Adamo DR, et al: Multicenter phase II trial of sunitinib in the treatment of nongastrointestinal stromal tumor sarcomas. J Clin Oncol 27: 3154-3160, 2009.

29. Stacchiotti S, Marrari A, Tamborini E, Palassini E, Virdis E, Messina A, Crippa F, Morosi C, Gronchi A, Pilotti S and Casali PG: Response to imatinib plus sirolimus in advanced chordoma. Ann Oncol 20: 1886-1894, 2009.

30. Meng T, Jin J, Jiang C, Huang R, Yin H, Song D and Cheng L: Molecular targeted therapy in the treatment of chordoma: A systematic review. Front Oncol 9: 30, 2019.

31. Liu C, Jia Q, Wei H, Yang X, Liu T, Zhao J, Ling Y, Wang C, Yu H, Li Z, et al: Apatinib in patients with advanced chordoma: A single-arm, single-centre, phase 2 study. Lancet Oncol 21: 1244-1252, 2020.

32. Stacchiotti S and Sommer J; Chordoma Global Consensus Group: Building a global consensus approach to chordoma: A position paper from the medical and patient community. Lancet Oncol 16: e71-e83, 2015. 
33. Stacchiotti S, Gronchi A, Fossati P, Akiyama T, Alapetite C, Baumann M, Blay JY, Bolle S, Boriani S, Bruzzi P, et al: Best practices for the management of local-regional recurrent chordoma: A position paper by the chordoma global consensus group. Ann Oncol 28: 1230-1242, 2017.

34. Colia V and Stacchiotti S: Medical treatment of advanced chordomas. Eur J Cancer 83: 220-228, 2017.

35. Ji T, Guo W, Yang R, Tang X, Wang Y and Huang L: What are the conditional survival and functional outcomes after surgical treatment of 115 patients with sacral chordoma? Clin Orthop Relat Res 475: 620-630, 2017.
36. Yang Y, Niu X, Li Y, Liu W and Xu H: Recurrence and survival factors analysis of 171 cases of sacral chordoma in a single institute. Eur Spine J 26: 1910-1916, 2017.

37. Zhou J, Sun J, Bai HX, Huang X, Zou Y, Tan X, Zhang Z, Tang X, Tao Y, Xiao B, et al: Prognostic factors in patients with spinal chordoma: An integrative analysis of 682 patients. Neurosurgery 81: 812-823, 2017.

This work is licensed under a Creative Commons Attribution-NonCommercial-NoDerivatives 4.0 International (CC BY-NC-ND 4.0) License. 